\title{
Excitonically driven quantum dot light-emitting diodes: exLEDs
}

\author{
Burak Guzelturk ${ }^{1}$, Pedro Ludwig Hernandez-Martinez ${ }^{1,2}$, Vijay Kumar Sharma ${ }^{1}$, Yasemin Coskun ${ }^{1}$, Vusala \\ Ibrahimova ${ }^{1}$, Xiao Wei Sun ${ }^{2}$, Donus Tuncel ${ }^{1}$, and Hilmi Volkan Demir ${ }^{1,2}$ \\ ${ }^{I}$ Department of Electrical and Electronics Engineering, Department of Physics, and UNAM - Institute of Materials Science and Nanotechnology \\ Bilkent University, Ankara, Turkey TR-06800 \\ ${ }^{2}$ LUMINOUS! Center of Excellence for Semiconductor Lighting and Displays, Microelectronics Division, School of Electrical and Electronics \\ Engineering and Physics and Applied Physics Division, School of Physical and Mathematical Sciences, Nanyang Technological University, \\ Nanyang Avenue, Singapore 639798, Singapore \\ Email: volkan@bilkent.edu.tr and hvdemir@ntu.edu.sg
}

\begin{abstract}
A hybrid platform of colloidal quantum dots integrated into conjugated polymers is reported for excitonically driven light-emitting diodes having pure quantum dot emission in the electroluminescence spectrum with substantially enhanced efficiency.

OCIS codes: (260.2160) Energy transfer; (250.2080) Polymer active devices; (250.5590) Quantum-well, -wire and -dot devices
\end{abstract}

\section{Introduction}

Semiconductor colloidal quantum dots (CQDs) are promising material systems for quality lighting and displays owing to their tunable emission properties with high photoluminescence quantum yields. CQD based light-emitting diodes (CQD-LEDs) essentially rely on charge injection pumping of the CQDs. In these devices, to facilitate charge injection, a very thin CQD film is employed in the active layer. [1] CQD thin films typically suffer from poor electrical transport properties due to their organic ligands. [2] Because of this limitation in CQD-LEDs, exciton recombination zone shifts with varying current injection levels, which is detrimental to the color stability and purity of these devices. [3] To address these obstructions of the charge injection into CQDs, here we propose the utilization of excitonic injection into CQDs replacing the common charge injection. To this end, we present a hybrid platform of CQDs that is developed to implement efficient excitonic injection in CQD-LEDs, which are dubbed as exLEDs here. [4] The proposed system allows for highly efficient excitonic operation due to specific hybridization between CQDs and the integrating polymer, thus enabling high CQD loadings. Consequently, nonradiative energy transfer (NRET) of the excitons can be maximized in the proposed hybrid CQD-based system.

\section{Principle of operation}

Excitonic operation of the exLEDs requires the separation of the emission and charge transport processes. For this purpose, carboxylic acid side-chain functionalized polyfluorene (PF) conjugated polymer is employed as the charge injection and exciton formation medium, while the core/alloyed-shell CdSe/CdS/ZnS CQDs are used as the lightemitting centers. Unlike previous reports on CQD-polymer blended systems, in this study, complete excitonic operation is accomplished to realize a new class of CQD-based excitonic LEDs. Formerly, epitaxially grown quantum well - CQD hybrid systems were proposed to operate in excitonic injection, but they were quite limited due to geometrical reasons [5,6], which are overcome in these exLEDs here. Since excitonic energy transfer through NRET is highly sensitive to average separation between donor-acceptor species, high CQD loadings has to be achieved in the host polymer, which serves as the exciton donor. Nevertheless, it is not trivial to reach high CQD loading levels in conjugated polymer hosts due to phase segregation phenomenon. That is why a functionalized PF, whose side chains have carboxyl acid functional groups to interact with CQD surfaces, is promoted in our hybrid material platform as shown in Fig. 1(a). The CQDs are more homogenously distributed in the functionalized polymer as shown in the transmission electron microscopy image in Fig. 1(b).

a


Fig. 1. (a) Illustration of the exciton drive from functionalized PF to different sized CQDs through nonradiative energy transfer. (b) Device architecture, shown along with transmission electron microscopy (TEM) image of the cross-section from the active layer (the scale bar is $50 \mathrm{~nm}$ ). 


\section{Experiments and Results}

Using this hybrid material platform, excitonically driven hybrid exLEDs were fabricated. The device employs a simple design of ITO/PEDOT:PSS/functionalized PF:CQDs/Al as shown in Fig. 1(b). Since this was a proof-ofconcept demonstration, we did not employ additional charge blocking layers, which would possibly make the devices more efficient. In Fig. 2(a) the electroluminescence (EL) spectra of exLEDs, which were fabricated using three different sized CQDs, are presented. ExLEDs exhibit complete and saturated CQD emission with completely quenched functionalized PF emission thanks to the highly efficient exciton transfer from the polymer into the CQDs. This total quenching of the polymer emission was confirmed to be dominantly due to the efficient NRET using timeresolved fluorescence spectroscopy. Furthermore, the charge transfer between the functionalized PF and CQDs is found to be not significant in our case, as also observed by Lutich et al. [7]

Generally, achieving pure CQD emission is not possible in LEDs using blended material systems of nonfunctionalized polymers and CQDs. To demonstrate this point, we also used non-functionalized PF with our CQDs as a reference blended material system. The resulting electroluminescence was always inferred with the emission of the non-functionalized PF (not shown here). This is attributed to the limited excitonic operation in the blended system, since CQDs are heavily aggregated inside the non-functionalized polymer at increased loadings. That is why homogenous films of high CQD loaded hybrids are essential to facilitating superior excitonic operation. Exciton injection-based exLEDs provide high color purity via suppressing the polymer emission owing to quite efficient exciton transfer as shown in Fig. 2(a). Fig. 2(b) presents the external quantum efficiencies (EQEs) of exLED and negative control group devices of only functionalized PF-LED and only CQD-LED. The exLED outperforms the control group devices by more than tenfold enhancement in the maximum EQEs. This demonstrates that excitonic injection is much more efficient than charge injection in the studied device architecture owing to the efficient exciton formation in the polymer and efficient exciton to photon conversion in the CQDs. Furthermore, these exLEDs have low turn on voltages $(<3.5 \mathrm{~V})$ and reach a maximum brightness level over $3000 \mathrm{~cd} / \mathrm{m}^{2}$ without an optimized structure.
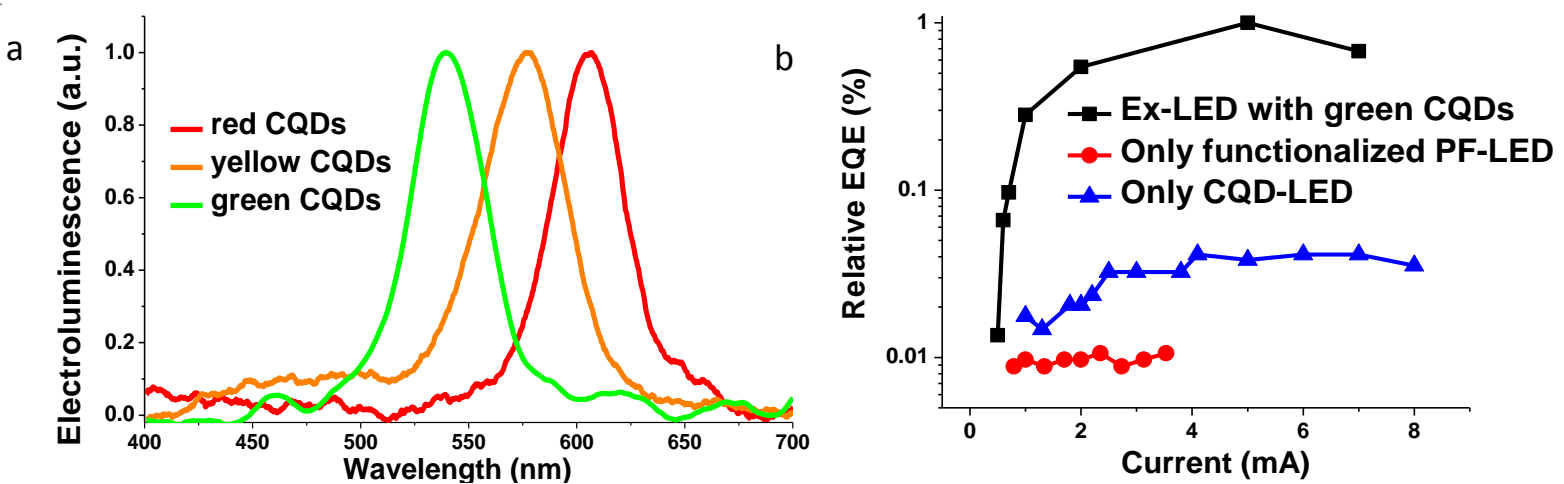

Fig. 2. (a) EL spectra of exLEDs with red-, yellow- and green-emitting CQDs. (b) Relative EQE of the exLED, only polymer-LED and onlyCQD LED.

In conclusion, we have developed a model material system of the colloidal CQDs and functionalized PF conjugated polymer to realize a hybrid medium, where efficient excitonic operation can be facilitated for CQD based LEDs. Here, we demonstrated proof-of-concept excitonically driven LEDs, which are promising for quality lighting and display applications.

This work is supported by National Research Foundation under Grant No. NRF-RF-2009-09 and NRF-CRP-6-2010-2. H.V.D. acknowledges support from ESF-EURYI and TUBA-GEBIP.

\section{References}

[1] S. Coe-Sullivan, W. Woo, M.G. Bawendi, V. Bulovic, Nature, 420, 800- 803 (2002).

[2] D. V. Talapin, C. B. Murray, Science, 310, 86-89 (2005).

[3] P. O. Anikeeva et al. Phys. Rev. B. 78, 085434 (2008).

[4] B. Guzelturk, P. L. M.Hernandez, Y. Coskun, V. Ibrahimova, D. Tuncel, X. W. Sun, H. V. Demir, (in submission)

[5] M. Achermann, M.A. Petruska, S. Kos, D. L. Smith, D. D. Koleske, V. I. Klimov, Nature 429, 642-646 (2004).

[6] S. Nizamoglu, B. Guzelturk, D.-W. Jeon, I.-H. Lee, H. V. Demir, App. Phys. Lett. 98, 163108, (2011).

[7] A. A. Lutich, G. Jiang, A. S. Susha, A. L. Rogach, F. D. Stefani, J. Feldmann, Nano Lett. 9, 2636-2640 (2009). 